\title{
Explicit asymptotic modelling of transient Love waves propagated along a thin coating
}

\author{
M. Ahmad, E. Nolde and A. V. Pichugin
}

\begin{abstract}
An explicit asymptotic model for transient Love waves is derived from the exact equations of anti-plane elasticity. The perturbation procedure relies upon the slow decay of low frequency Love waves to approximate the displacement field in the substrate by a power series in the depth coordinate. When appropriate decay conditions are imposed on the series, one obtains a model equation governing the displacement at the interface between the coating and the substrate. Unusually, the model equation contains a term with a pseudo-differential operator. This result is confirmed and interpreted by analysing the exact solution obtained by integral transforms. The performance of the derived model is illustrated by numerical examples.
\end{abstract}

Keywords. asymptotics, long wave, coating, Love waves.

\section{Introduction}

A. E. H. Love [15] gave the first example of a surface wave guided by a subsurface inhomogeneity. Motivated by the anti-plane problem of 2D elasticity, Love's original contribution focussed on the shear horizontal (SH) waves guided by a layer bonded to a half-space. In contrast to the (non-dispersive) Rayleigh waves, the resulting solutions were shown to form an infinite spectrum of dispersive modes propagating in a fixed range of phase velocities. Subsequently, these results were generalised to multiple and/or periodic layers of anisotropic and functionally-graded materials, see $[9,3,8,22]$ and references therein.

The research on Love waves and their generalisations has for a long time been driven by applications to geophysics and ocean acoustics. This motivated particular interest in the exact solutions of transient problems for sub-surface sources and complex inhomogeneity profiles. For instance, the excitation of Love waves by sub-surface sources was analysed in [19, 18]. Much has been 
written on the closely related problems in ocean acoustics, concerning the transient response of Pekeris' waveguide [17, 5, 7].

If one assumes that the solution is dominated by the low frequencies or, equivalently, that a dominant wavelength greatly exceeds the thickness of the coating layer, then the problem offers dramatic simplifications due to the quasi-static conditions governing the field within the coating. The dispersion of Love waves in this asymptotic regime has been studied in the works $[20,21,23]$. It is worth remarking that the low-frequency asymptotics are less relevant in ocean acoustics, because Pekeris' waveguide does not have a fundamental mode.

An elegant way to make use of the simplifications offered by the low frequency regime in the presence of external forces originates from the work of Achenbach, Keshava and Tiersten, see [2, 24], who showed that the response of a thin coating can be reproduced by asymptotically equivalent effective boundary condition(s), see also $[4,25,6]$. This methodology was recently extended to develop explicit models for surface waves. In these models, solutions of partial differential equations describing the evolution of the surface wave serve as boundary conditions for the field in substrate $[1,12,16,14]$.

This paper is devoted to the analysis of the transient surface excitation problem for SH Love waves in the limit of vanishing thickness of the coating layer. The perturbation procedure implemented is novel in that it relies upon the slow decay of low frequency Love waves to approximate the field in the substrate by a power series in terms of the depth coordinate. The associated asymptotic model is then generated by imposing an appropriate behaviour of the displacement field at infinity. Unusually, the resulting low frequency model for Love waves features a term with a pseudo-differential operator. The exact integral transform solution of the problem is then studied to analyse the significance of this result. The performance of the derived model is illustrated by several numerical examples.

\section{Statement of the problem}

Consider an isotropic elastic layer of thickness $H$, bonded at the interface $y=0$ to the substrate of another isotropic elastic material occupying the upper half-space $y>0$, see Figure 1 . The propagation of $\mathrm{SH}$ waves in this setting is governed by the anti-plane equations of motion

$$
\begin{aligned}
\frac{\partial^{2} u_{l}}{\partial x^{2}}+\frac{\partial^{2} u_{l}}{\partial y^{2}} & =\frac{1}{c_{l}^{2}} \frac{\partial^{2} u_{l}}{\partial t^{2}}, \\
\frac{\partial^{2} u_{s}}{\partial x^{2}}+\frac{\partial^{2} u_{s}}{\partial y^{2}} & =\frac{1}{c_{s}^{2}} \frac{\partial^{2} u_{s}}{\partial t^{2}},
\end{aligned}
$$

where $u=u(x, y, t)$ is the out-of-plane displacement, $c \equiv \sqrt{\mu / \rho}$ the shear wave speed, $\mu$ the shear modulus and $\rho$ the density. The subscripts " $l$ " and " $s$ " are used to denote quantities related to the layer and the substrate, respectively. 


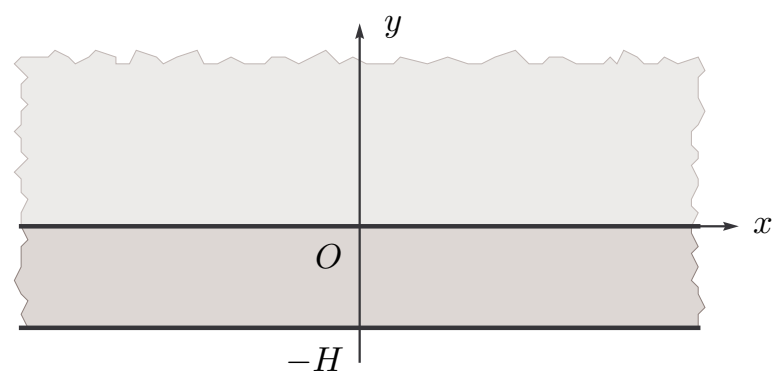

Figure 1. The coordinate system used in the paper.

Love waves are harmonic plane wave solutions of the equations (1)-(2) that satisfy the homogeneous boundary conditions

$$
\left.\frac{\partial u_{l}}{\partial y}\right|_{y=-H}=0,\left.\quad u_{l}\right|_{y=0}=\left.u_{s}\right|_{y=0},\left.\quad \mu_{l} \frac{\partial u_{l}}{\partial y}\right|_{y=0}=\left.\mu_{s} \frac{\partial u_{s}}{\partial y}\right|_{y=0},
$$

and are localised near the surface, i.e. decay exponentially in the substrate. It is readily verified that the appropriate solutions are of the following form

$$
\left(\begin{array}{l}
u_{l} \\
u_{s}
\end{array}\right)=C\left(\begin{array}{c}
\cos \left(p_{l} k(y+H)\right) \\
\cos \left(p_{l} k H\right) \exp \left(-p_{s} k y\right)
\end{array}\right) \mathrm{e}^{\mathrm{i} k(x-v t),},
$$

where $k$ is the wave number, $p_{l}=\sqrt{\chi^{2} v^{2} / c_{s}^{2}-1}$ and $p_{s}=\sqrt{1-v^{2} / c_{s}^{2}}$. The phase velocity $v, c_{l}<v<c_{s}$, must then satisfy the Love secular equation

$$
\tan \left(p_{l} k H\right)=\gamma \frac{p_{s}}{p_{l}} .
$$

The non-dimensional ratios of characteristic velocities and shear modulae, $\chi$ and $\gamma$, that appear within (4)-(5) are defined by

$$
\chi=\frac{c_{s}}{c_{l}}, \quad \gamma=\frac{\mu_{s}}{\mu_{l}} .
$$

The implicit relation (5) describes the dispersion of Love waves. Our main focus in this paper is on those Love waves whose typical wavelength $\lambda \equiv 1 / k$ is much larger than the thickness of the coating layer $H$. Thus, our problem possesses a natural small parameter $\eta \equiv H / \lambda=k H \ll 1$. The relation (5) indicates that when $\eta \rightarrow 0$, we have $p_{s} \rightarrow 0$, hence $v / c_{s} \sim 1$. Consequently, the appropriately rescaled frequency $\bar{\omega} \equiv \omega H / c_{s}=v k H / c_{s} \sim \eta$ must also be low. Further expansion of (5) yields

$$
\eta=\bar{\omega}+\frac{\left(\chi^{2}-1\right)^{2}}{2 \gamma^{2}} \bar{\omega}^{3}+O\left(\bar{\omega}^{5}\right) .
$$

This long wave limit may also be referred to as the low frequency limit.

The rest of the paper is concerned with the solution of inhomogeneous problem, in which condition $(3)_{1}$ is replaced by

$$
\left.\mu_{l} \frac{\partial u_{l}}{\partial y}\right|_{y=-H}=P
$$


with $P=P(x, t)$ denoting the load on the surface of the coating. In principle, this problem can be solved explicitly, for example, by the application of Green's function that is available in this case [7]. Nevertheless, our main interest here is in the development of asymptotic methodologies that can be applied to problems where no explicit Green's function is available, see e.g. [13].

\section{Transient Love waves in a thin coating}

Consider the situation when the wavelength $\lambda$ dominating the dynamic response is much longer than the coating thickness $H$. We begin the asymptotic analysis of this problem by appropriately non-dimensionalising the governing equations (1)-(2) and the boundary conditions $(3)_{2,3}$ and (8). The space and time coordinates are rescaled according to

$$
x=\lambda \xi, \quad y=H \zeta, \quad t=\frac{\lambda}{c_{s}} \tau .
$$

The appropriate displacement and stress fields are rescaled as

$$
u_{l}=\lambda u_{l}^{*}, \quad u_{s}=\lambda u_{s}^{*}, \quad P=\eta \mu_{s} P^{*},
$$

with asterisks denoting non-dimensional quantities. The resulting boundary value problem is then given by

$$
\begin{gathered}
\frac{\partial^{2} u_{l}^{*}}{\partial \zeta^{2}}+\eta^{2} \mathcal{J}\left[u_{l}^{*}\right]=0, \quad \frac{\partial^{2} u_{s}^{*}}{\partial \zeta^{2}}-\eta^{4} \mathcal{L}\left[u_{s}^{*}\right]=0 \\
\left.\frac{\partial u_{l}^{*}}{\partial \zeta}\right|_{\zeta=-1}=\gamma \eta^{2} P^{*},\left.\quad u_{l}^{*}\right|_{\zeta=0}=\left.u_{s}^{*}\right|_{\zeta=0},\left.\quad \frac{\partial u_{l}^{*}}{\partial \zeta}\right|_{\zeta=0}=\left.\gamma \frac{\partial u_{s}^{*}}{\partial \zeta}\right|_{\zeta=0},
\end{gathered}
$$

in which we have introduced the operators

$$
\mathcal{J} \equiv \frac{\partial^{2}}{\partial \xi^{2}}-\chi^{2} \frac{\partial^{2}}{\partial \tau^{2}} \quad \text { and } \quad \mathcal{L} \equiv \eta^{-2}\left(\frac{\partial^{2}}{\partial \tau^{2}}-\frac{\partial^{2}}{\partial \xi^{2}}\right)
$$

such that $\mathcal{J}\left[u_{l}^{*}\right] \sim u_{l}^{*}$ and $\mathcal{L}\left[u_{s}^{*}\right] \sim u_{s}^{*}$. The fourth boundary condition for this problem is furnished by requiring that the displacement field $u_{s}^{*}$ is generated solely by the surface source in $(12)_{1}$ and, consequently, decays as $\zeta \rightarrow \infty$.

The solution is sought in the form of the ansatz

$$
\begin{aligned}
& u_{l}^{*}(\xi, \zeta, \tau)=u_{l}^{(0)}+\eta^{2} u_{l}^{(2)}+\eta^{4} u_{l}^{(4)}+\ldots \\
& u_{s}^{*}(\xi, \zeta, \tau)=u_{s}^{(0)}+\eta^{2} u_{s}^{(2)}+\eta^{4} u_{s}^{(4)}+\ldots
\end{aligned}
$$

where $u^{(i)}=u^{(i)}(\xi, \zeta, \tau)$, with superscripts indicating the asymptotic orders of the associated functions. Upon substituting this ansatz into the boundary value problem (11)-(12) we end up with a hierarchy of boundary value problems for various orders of $\eta$. 
The leading-order problem is given by

$$
\begin{gathered}
\frac{\partial^{2} u_{l}^{(0)}}{\partial \zeta^{2}}=0, \quad \frac{\partial^{2} u_{s}^{(0)}}{\partial \zeta^{2}}=0 \\
\left.\frac{\partial u_{l}^{(0)}}{\partial \zeta}\right|_{\zeta=-1}=0,\left.\quad u_{l}^{(0)}\right|_{\zeta=0}=\left.u_{s}^{(0)}\right|_{\zeta=0},\left.\quad \frac{\partial u_{l}^{(0)}}{\partial \zeta}\right|_{\zeta=0}=\left.\gamma \frac{\partial u_{s}^{(0)}}{\partial \zeta}\right|_{\zeta=0} .
\end{gathered}
$$

It is easily verified that the solutions of the equations (16) satisfying the boundary conditions $(17)_{1,3}$ can be written as

$$
u_{l}^{(0)}=U_{l}^{(0,0)}(\xi, \tau), \quad u_{s}^{(0)}=U_{s}^{(0,0)}(\xi, \tau) .
$$

Here and henceforth we shall use double superscripts to indicate the quantities independent of the normal coordinate $\zeta$. The remaining boundary condition $(17)_{2}$ requires that

$$
U_{l}^{(0,0)}=U_{s}^{(0,0)} .
$$

The leading order solution is now presented in terms of the function $U_{s}^{(0,0)}$, which is unspecified as of yet. This is the usual situation in applications of asymptotic integration, see e.g. [13]. We expect to be able to identify this function by considering boundary value problems of higher orders and taking into account (still unspecified) conditions of decay at infinity.

The problem for the next order has the form

$$
\begin{gathered}
\frac{\partial^{2} u_{l}^{(2)}}{\partial \zeta^{2}}=-\mathcal{J}\left[U_{s}^{(0,0)}\right], \quad \frac{\partial^{2} u_{s}^{(2)}}{\partial \zeta^{2}}=0, \\
\left.\frac{\partial u_{l}^{(2)}}{\partial \zeta}\right|_{\zeta=-1}=\gamma P^{*},\left.\quad u_{l}^{(2)}\right|_{\zeta=0}=\left.u_{s}^{(2)}\right|_{\zeta=0},\left.\quad \frac{\partial u_{l}^{(2)}}{\partial \zeta}\right|_{\zeta=0}=\left.\gamma \frac{\partial u_{s}^{(2)}}{\partial \zeta}\right|_{\zeta=0} .
\end{gathered}
$$

The general solution of equations (20) may be written as

$$
u_{l}^{(2)}=-\frac{1}{2} \mathcal{J}\left[U_{s}^{(0,0)}\right] \zeta^{2}+U_{l}^{(2,1)} \zeta+U_{l}^{(2,0)}, \quad u_{s}^{(2)}=U_{s}^{(2,1)} \zeta+U_{s}^{(2,0)} .
$$

Upon invoking boundary conditions (21), most unknown quantities within (22) can be expressed in terms of $U_{s}^{(0,0)}$ and $P^{*}$, which yields

$$
\begin{gathered}
U_{l}^{(2,1)}=-\mathcal{J}\left[U_{s}^{(0,0)}\right]+\gamma P^{*}, \quad U_{l}^{(2,0)}=U_{s}^{(2,0)}, \\
U_{s}^{(2,1)}=-\gamma^{-1} \mathcal{J}\left[U_{s}^{(0,0)}\right]+P^{*} .
\end{gathered}
$$

It is worth noting that the leading order displacement $U_{s}^{(0,0)}$ remains unspecified, as well as the newly introduced displacement function $U_{s}^{(2,0)}$. To determine these functions one needs to consider higher order problems.

The second order boundary value problem is given by

$$
\begin{gathered}
\frac{\partial^{2} u_{l}^{(4)}}{\partial \zeta^{2}}=-\mathcal{J}\left[u_{l}^{(2)}\right], \quad \frac{\partial^{2} u_{s}^{(4)}}{\partial \zeta^{2}}=\mathcal{L}\left[u_{s}^{(0)}\right] \\
\left.\frac{\partial u_{l}^{(4)}}{\partial \zeta}\right|_{\zeta=-1}=0,\left.\quad u_{l}^{(4)}\right|_{\zeta=0}=\left.u_{s}^{(4)}\right|_{\zeta=0},\left.\quad \frac{\partial u_{l}^{(4)}}{\partial \zeta}\right|_{\zeta=0}=\left.\gamma \frac{\partial u_{s}^{(4)}}{\partial \zeta}\right|_{\zeta=0} .
\end{gathered}
$$


The solution of this problem can be written in the form of polynomials in $\zeta$

$$
u_{l}^{(4)}=\sum_{i=0}^{4} U_{l}^{(4, i)} \zeta^{i}, \quad u_{s}^{(4)}=\sum_{i=0}^{2} U_{s}^{(4, i)} \zeta^{i},
$$

where

$$
\begin{gathered}
U_{l}^{(4,4)}=\frac{1}{24} \mathcal{J}^{2}\left[U_{s}^{(0,0)}\right], \quad U_{l}^{(4,3)}=\frac{1}{6} \mathcal{J}^{2}\left[U_{s}^{(0,0)}\right]-\frac{1}{6} \gamma \mathcal{J}\left[P^{*}\right], \\
U_{l}^{(4,2)}=-\frac{1}{2} \mathcal{J}\left[U_{s}^{(2,0)}\right], \quad U_{s}^{(4,2)}=\frac{1}{2} \mathcal{L}\left[U_{s}^{(0,0)}\right] .
\end{gathered}
$$

The remaining quantities with a double superscript are found from boundary conditions (21), with the result

$$
\begin{gathered}
U_{l}^{(4,1)}=-\frac{1}{3} \mathcal{J}^{2}\left[U_{s}^{(0,0)}\right]+\frac{1}{2} \gamma \mathcal{J}\left[P^{*}\right]-\mathcal{J}\left[U_{s}^{(2,0)}\right], \\
U_{s}^{(4,1)}=\gamma^{-1} U_{l}^{(4,1)}, \quad U_{l}^{(4,0)}=U_{s}^{(4,0)} .
\end{gathered}
$$

Thus, the functions $U_{l}^{(4, i)}$ and $U_{s}^{(4, j)}(i=\overline{1,4}, j=\overline{0,2})$ have now all been expressed in terms of $U_{s}^{(0,0)}$ and $U_{s}^{(2,0)}$. At the same time yet another unspecified function $U_{s}^{(4,0)}$ has arisen.

The solutions of the boundary value problems considered thus far may be combined, with the resulting expansion for $u_{s}^{*}$ of the form

$$
\begin{aligned}
u_{s}^{*}=\{ & \left.U_{s}^{(0,0)}+\eta^{2} U_{s}^{(2,1)} \zeta+\eta^{4} U_{s}^{(4,2)} \zeta^{2}\right\} \\
& +\eta^{2}\left(U_{s}^{(2,0)}+\eta^{2} U_{s}^{(4,1)} \zeta\right)+\eta^{4} U_{s}^{(4,0)}+\ldots
\end{aligned}
$$

Solutions that satisfy the decay conditions at infinity must behave as an exponential decaying as $\zeta \rightarrow \infty$. Note that all the quantities within the braces only depend upon the unknown function $U_{s}^{(0,0)}$, but do not depend upon $U_{s}^{(2,0)}$ or $U_{s}^{(4,0)}$. Therefore, we observe that the decay conditions may be satisfied to $O\left(\eta^{2}\right)$ by requiring that the terms in braces match the first three terms in the formal series expansion of $\exp \left(-\eta^{2} \zeta \mathcal{F}\right) U_{s}^{(0,0)}$, where $\mathcal{F}$ is a pseudo-differential operator to be determined. The resulting matching procedure requires one to satisfy the two relations

$$
\mathcal{F}^{2}\left[U_{s}^{(0,0)}\right]=\mathcal{L}\left[U_{s}^{(0,0)}\right], \quad \mathcal{F}\left[U_{s}^{(0,0)}\right]-\gamma^{-1} \mathcal{J}\left[U_{s}^{(0,0)}\right]+P^{*}=0 .
$$

From $(30)_{1}$ we conclude that $\mathcal{F}=\mathcal{L}^{\frac{1}{2}}$, so that $(30)_{2}$ leads to the pseudodifferential governing equation for the long wave displacement $U_{s}^{(0,0)}$ given by

$$
\mathcal{L}^{\frac{1}{2}}\left[U_{s}^{(0,0)}\right]-\gamma^{-1} \mathcal{J}\left[U_{s}^{(0,0)}\right]=-P^{*} .
$$

It is worth remarking that the more accurate counterpart of (31), as well as the governing equation for $U_{s}^{(2,0)}$, may be obtained by enforcing the decay condition on the next order solution.

The long wave governing equation (31) describes the displacement at the interface between the layer and the substrate as a function of the surface loading. Hence, the solutions of initial/boundary value problems for a 
half-space with a thin coating can be found to within $O\left(\eta^{2}\right)$ by solving the equation (31) subject to appropriate initial conditions. The associated displacement fields can then be recovered using (14)-(15) and (22).

In the absence of the surface loads equation (31) can be satisfied by plane wave solutions $U_{s}^{(0,0)}=U e^{\mathrm{i}(k x-\omega t)} \equiv U e^{\mathrm{i}(\xi-\bar{\omega} \tau / \eta)}$, where we have re-used some of the notation from Section 2. By inserting these solutions into (31) and re-expanding the result for small $\bar{\omega}$ one obtains the approximate secular equation (7) exactly, hence confirming the consistency of our approach.

\section{Analysis of the exact solution}

The described perturbation method does not provide any estimate for the range of applicability of the governing equation (31). In order to better understand the assumptions underlying our low frequency model, the exact integral transform solution of the original two-dimensional problem is constructed and analysed in this Section. This analysis will also provide an alternative demonstration of validity of the equation (31) and help in interpreting the physical significance of the term with the pseudo-differential operator appearing in (31).

Suppose that the (non-dimensional) boundary value problem (11), (12) is solved subject to zero initial conditions. An application of the Fourier transform with respect to longitudinal coordinate $\xi$, followed by an application of the Fourier transform with respect to time $\tau$, leads to the system of ordinary differential equations

$$
\frac{\partial^{2} u_{l}^{F}}{\partial \zeta^{2}}+q_{l}^{2} \eta^{2} u_{l}^{F}=0, \quad \frac{\partial^{2} u_{s}^{F}}{\partial \zeta^{2}}-q_{s}^{2} \eta^{2} u_{s}^{F}=0,
$$

with $q_{l}^{2} \equiv \chi^{2} \Omega^{2}-\kappa^{2}$ and $q_{s}^{2} \equiv \kappa^{2}-\Omega^{2}$; which must be solved subject to the inhomogeneous boundary conditions

$$
\left.\frac{\partial u_{l}^{F}}{\partial \zeta}\right|_{\zeta=-1}=\gamma \eta^{2} P_{F},\left.\quad u_{l}^{F}\right|_{\zeta=0}=\left.u_{s}^{F}\right|_{\zeta=0},\left.\quad \frac{\partial u_{l}^{F}}{\partial \zeta}\right|_{\zeta=0}=\left.\gamma \frac{\partial u_{s}^{F}}{\partial \zeta}\right|_{\zeta=0},
$$

where

$$
P_{F}(\kappa, \Omega)=\int_{-\infty}^{\infty} \int_{-\infty}^{\infty} P^{*}(\xi, \tau) e^{-\mathrm{i}(\kappa \xi-\Omega \tau)} \mathrm{d} \tau \mathrm{d} \xi
$$

This problem can be solved explicitly and, in particular, gives a simple expression for the transformed displacement field in the substrate,

$$
u_{s}^{F}=-\frac{\eta P_{F} e^{-q_{s} \eta \zeta}}{q_{s} \cos \left(q_{l} \eta\right)-\gamma^{-1} q_{l} \sin \left(q_{l} \eta\right)},
$$

which immediately leads to the exact formal solution for the displacement

$$
u_{s}(\xi, \zeta, \tau)=-\frac{1}{4 \pi^{2}} \int_{-\infty}^{\infty} \int_{-\infty}^{\infty} \frac{\eta P_{F} e^{-q_{s} \eta \zeta}}{q_{s} \cos \left(q_{l} \eta\right)-\gamma^{-1} q_{l} \sin \left(q_{l} \eta\right)} e^{\mathrm{i}(\kappa \xi-\Omega \tau)} \mathrm{d} \Omega \mathrm{d} \kappa .
$$

Note that we fixed the branch of the radical implicit in the definition of parameter $q_{s}$ by requiring that $\Re\left(q_{s}\right)>0$. A similar expression can be obtained 


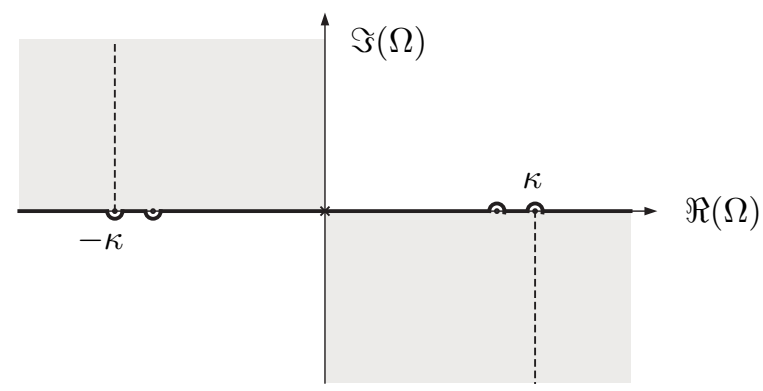

Figure 2. The choice of branch cuts and integration contour in complex $\Omega$ plane for the inner Fourier integral in (36).

for displacement within the layer, however, it is omitted here for the sake of brevity. Henceforth, we shall assume that $\kappa$ is real and that we are working in the complex $\Omega$ plane. Since we are interested in the far field (long time) solutions at the interface $\zeta=0$, the decay of the right-propagating waves at $\tau \rightarrow \infty$ requires that $\Im(\Omega) \leqslant 0$. Similar argument applied to left-propagating waves leads to the conclusion that the $\Omega$-integration contour must be confined to the second and fourth quadrants. A standard configuration of branch cuts and integration contour for this type of problem is illustrated in Figure 2; a detailed discussion of this choice is provided in [10] or, for a slightly different problem of transient excitation of Pekeris' waveguide, in [7].

For every fixed value of $\kappa$ the transform solution (35) has a number of singularities in complex $\Omega$ plane. These include one (or several) pairs of real poles corresponding to Love wave modes; branch cuts emanating from the branch points $\Omega_{s}= \pm \kappa$, associated with the wave front propagating in the substrate; as well as an infinite number of complex poles deep in the second and fourth quadrants. The relative contributions of these singularities are, to a large extent, determined by the function $P_{F}$ characterising the spectral density of surface loading, see (34). The assumption that the coating layer is thin is equivalent to saying that the parameter $\eta$ is small in (35), so for a $P_{F}$ that decays sufficiently rapidly as $|\Omega| \rightarrow \infty$ and $|\kappa| \rightarrow \infty$, the response can be dominated by the low frequency (low wave number) singularities of the integrand. Henceforth, we assume that $P_{F} \rightarrow 0$ as $|\Omega| \rightarrow \infty$ and $|\kappa| \rightarrow \infty$ in such way that the denominator of (35) can be replaced by the first two terms in its Maclauren series expansion in $\eta$. The resulting approximation may be written as

$$
u_{s}^{F} \approx-\frac{P_{F} e^{-q_{s} \eta \zeta}}{q_{s} / \eta-\gamma^{-1} q_{l}^{2}} .
$$

If we specialise (37) to determine displacement field at the interface $\zeta=$ 0 , then it is immediately clear that approximation (37) is fully equivalent to pseudo-differential equation (31). The response associated with (37) is dominated by contribution of the poles corresponding to the fundamental Love wave mode, as well as by the contribution of adjacent branch cuts, 


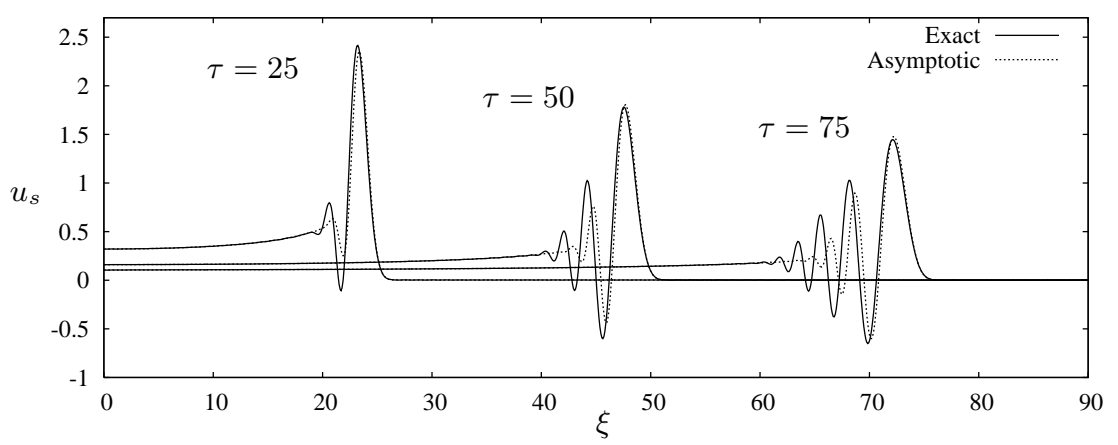

Figure 3. Displacement at the interface $\left.u_{s}\right|_{\zeta=0}$ produced by the loading (38) is evaluated at three instants of time: $\tau=25, \tau=50$ and $\tau=75$, where $\eta=0.5, \chi=1.2$ and $\gamma=$ 1.5. Exact and asymptotic solutions corresponding to (35) and (37) are plotted as solid and dashed lines, respectively.

see Figure 2. When modelling non-dispersive surface waves in the far field, one can often neglect the rapidly decaying contributions of the branch-cuts related to the wave front, see e.g. [11, 12]. The vicinity of the Love wave poles to the branch points precludes this possibility in our problem and explains the necessity of having a pseudo-differential operator in the governing equation.

The applicability of the approximation (37) may be analysed more precisely by specifying an explicit form for the function $P_{F}$. When the applied loading is an appropriately scaled Gaussian distribution with respect to both time and space, $P_{F}$ takes a particularly simple form

$$
P^{*}=M \frac{\exp \left[-\left(\xi^{2}+\tau^{2}\right) / 4 a^{2}\right]}{4 \pi a^{2}}, \quad \text { so } \quad P_{F}=M \exp \left[-a^{2}\left(\kappa^{2}+\Omega^{2}\right)\right],
$$

with the non-dimensional magnitude $M$ and the parameter $a$ characterising the degree of load concentration. The second pair of real poles in the denominator of (35), omitted in the approximation (37), appears when $q_{s}$ and $\sin \left(q_{l} \eta\right)$ vanish simultaneously. This happens first when

$$
\Omega=\kappa=\frac{\pi}{2 \eta \sqrt{\chi^{2}-1}} .
$$

By assuming that the material contrast is $O(1)$, the contributions of the second (and higher) pairs of real poles are negligible for the loading (38) as long as $a^{2} / \eta^{2} \gg 1$.

The presented analysis was supported by brute-force numerical computations of the integral (36). The load on the surface of the coating layer was chosen in the form (38) with $M=100$ and $a=1 / 3$. The snapshots of the displacement at the interface between the layer and the substrate were then computed for several fixed instants of time. In our computations, the 


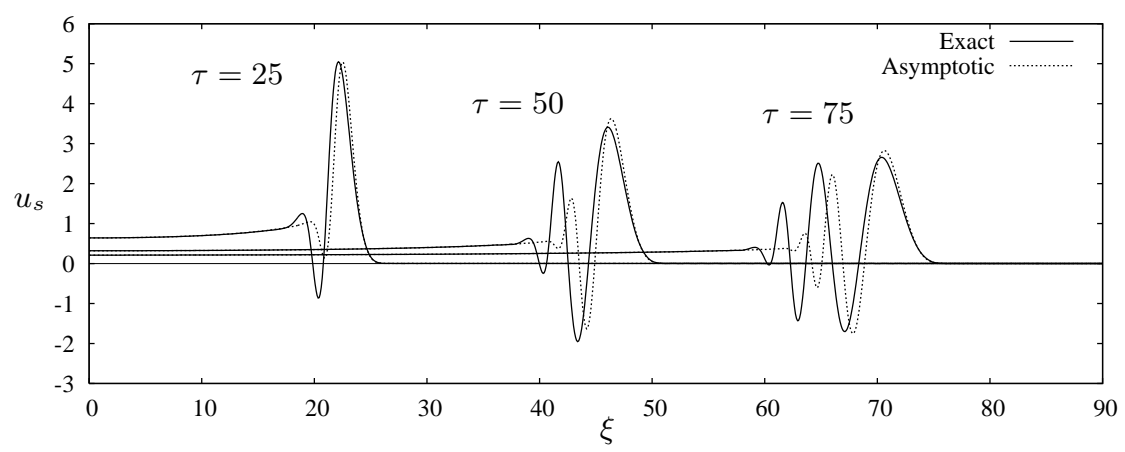

Figure 4. Displacement at the interface $\left.u_{s}\right|_{\zeta=0}$ produced by the loading (38) is evaluated at three instants of time: $\tau=25, \tau=50$ and $\tau=75$, where $\eta=1.0, \chi=1.2$ and $\gamma=$ 1.5. Exact and asymptotic solutions corresponding to (35) and (37) are plotted as solid and dashed lines, respectively.

conservative choices for $\eta$, which ensured that $a^{2} / \eta^{2} \gg 1$, resulted in excellent correspondence between the exact and approximate solutions, to the point that their plots were indistinguishable. Therefore, we felt justified in selecting relatively large values of $\eta$ capable of exposing the deficiencies of the approximate model.

The traces in Figure 3 show the evolution of the original Gaussian pulse as it propagates away from the loading point along the half-space coated by a relatively thin layer. The marked non-symmetry of the travelling disturbance is explained by two effects: the low-frequency dispersion of the Love fundamental mode, which results in the oscillations trailing the main pulse, and the contribution of the wave front, which caused the long tail of the disturbance. The principal deficiency of the approximate model manifests itself as a phase error best seen in the oscillatory trail of the propagating Gaussian. As the relative thickness of the coating grows, the inaccuracies involved in using (37) (or, equivalently, (31)) increase, so that both phase and magnitude of the propagated disturbance become distorted. This is best evidenced by Figure 4 computed for a thicker coating.

\section{Acknowledgement}

The paper was written while MA was visiting Brunel University, sponsored by the grant from Higher Education of Pakistan. The work of AVP was supported by Brunel University's "BRIEF" research award. Both of these awards are gratefully acknowledged. The paper greatly benefited from the discussions with Julius Kaplunov (Brunel University). 


\section{References}

[1] J. D. Achenbach. Explicit solutions for carrier waves supporting surface waves and plate waves. Wave Motion, 28(1):89-97, 1998.

[2] J. D. Achenbach and S. P. Keshava. Free waves in a plate supported by a semi-infinite continuum. Journal of Applied Mechanics, 34:397-404, 1967.

[3] K. Aki and P. G. Richards. Quantitative Seismology. W. H. Freeman \& Co, San Francisco, 1980.

[4] B. A. Auld. Acoustic Fields and Waves in Solids, volume 2. Krieger Pub. Co, Malabar, FL, 2nd edition, 1990.

[5] L. M. Brekhovskikh. Waves in Layered Media. Academic Press, New York, 1980.

[6] H.-H. Dai, J. Kaplunov, and D. A. Prikazchikov. A long wave model for the surface elastic wave in a coated half-space. Proceedings of the Royal Society of London, Series A, 2010. Published online.

[7] J. A. DeSanto. Scalar Wave Theory. Springer-Verlag, New York, 1992.

[8] E. H. El Boudouti, B. Djafari-Rouhani, A. Akjouj, and L. Dobrzynski. Theory of surface and interface transverse elastic waves in $n$-layer superlattices. Physical Review B, 54(20):14728-14741, 1996.

[9] W. M. Ewing, W. S. Jardetzky, and F. Press. Elastic Waves in Layered Media. McGraw-Hill, New York, 1957.

[10] J. G. Harris. Linear Elastic Waves. Cambridge University Press, Cambridge, 2001.

[11] J. Kaplunov, L. Kossovich, and A. Zakharov. An explicit asymptotic model for the Bleustein-Gulyaev wave. Comptes Rendus Mecanique, 332(7):487-492, 2004 .

[12] J. Kaplunov, A. Zakharov, and D. Prikazchikov. Explicit models for elastic and piezoelastic surface waves. IMA Journal of Applied Mathematics, 71(5):768$782,2006$.

[13] J. D. Kaplunov, L. Yu. Kossovich, and E. V. Nolde. Dynamics of Thin Walled Elastic Bodies. Academic Press, New York, 1998.

[14] A. P. Kiselev and D. F. Parker. Omni-directional Rayleigh, Stoneley and Schölte waves with general time dependence. Proceedings of the Royal Society of London, Series A, 2010. Published online.

[15] A. E. H. Love. Some Problems of Geodynamics. Cambridge University Press, London, 1911.

[16] D. F. Parker and A. P. Kiselev. Rayleigh waves having generalised lateral dependence. The Quarterly Journal of Mechanics and Applied Mathematics, 62(1):19-30, 2009.

[17] C. L. Pekeris. Theory of propagation of explosive sound in shallow water. In Propagation of Sound in the Ocean, The Geological Society of America, Memoir 27. 1948.

[18] Y. Satô. Study on surface waves. VI. Generation of Love and other type of SH waves. Bulletin of the Earthquake Research Institute (Tokyo), 30:101-120, 1952 .

[19] K. Sezawa. Love-waves generated from a source of a certain depth. Bulletin of the Earthquake Research Institute (Tokyo), 13:1-17, 1935. 
[20] A. L. Shuvalov and A. G. Every. Some properties of surface acoustic waves in anisotropic-coated solids, studied by the impedance method. Wave Motion, 36(3):257-273, 2002.

[21] A. L. Shuvalov and A. G. Every. On the long-wave onset of dispersion of the surface-wave velocity in coated solids. Wave Motion, 45(6):857-863, 2008.

[22] A. L. Shuvalov, O. Poncelet, and A. N. Podlipenets. On the guided and surface shear horizontal waves in monoclinic transversely periodic layers and halfspaces with arbitrary variation of material properties across the unit cell. Studia Geophysica et Geodaetica, 50(3):381-398, 2006.

[23] D. J. Steigmann and R. W. Ogden. Surface waves supported by thinfilm/substrate interactions. IMA Journal of Applied Mathematics, 72(6):730$747,2007$.

[24] H. F. Tiersten. Elastic surface waves guided by thin films. Journal of Applied Physics, 40(2):770-789, 1969.

[25] D. D. Zakharov. High order approximate low frequency theory of elastic anisotropic lining and coating. The Journal of the Acoustical Society of America, 119(4):1961-1970, 2006.

\author{
M. Ahmad \\ Department of Mathematics, \\ University of Sargodha, \\ Sargodha, Pakistan \\ e-mail: amasud112@yahoo.com \\ E. Nolde \\ Department of Mathematical Sciences, \\ Brunel University, \\ Uxbridge UB8 3PH, UK \\ e-mail: evgeniya.nolde@brunel.ac.uk
}
A. V. Pichugin
Department of Mathematical Sciences,
Brunel University,
Uxbridge UB8 3PH, UK
e-mail: aleksey.pichugin@brunel.ac.uk 